\title{
The posteromedial corner of the knee: an international expert consensus statement on diagnosis, classification, treatment, and rehabilitation
}

\author{
Jorge Chahla ${ }^{1}$ - Kyle N. Kunze ${ }^{2} \cdot$ Robert F. LaPrade $^{3} \cdot$ Alan Getgood $^{4} \cdot$ Moises Cohen $^{5} \cdot$ Pablo Gelber $^{6,7}$. \\ Björn Barenius $^{8}$. Nicolas Pujol ${ }^{9,10}$. Manual Leyes ${ }^{11} \cdot$ Ralph Akoto $^{12} \cdot$ Brett Fritsch $^{13}$. Fabrizio Margheritini ${ }^{14}$. \\ Leho Rips $^{15}$. Jakub Kautzner ${ }^{16}$. Victoria Duthon ${ }^{17}$. Danilo Togninalli ${ }^{18}$. Zanon Giacamo ${ }^{19}$. Nicolas Graveleau ${ }^{20}$. \\ Stefano Zaffagnini ${ }^{21} \cdot$ Lars Engbretsen $^{22} \cdot$ Martin Lind $^{23} \cdot$ Rodrigo Maestu $^{24} \cdot$ Richard Von Bormann $^{25}$. \\ Charles Brown ${ }^{26}$. Silvio Villascusa ${ }^{27}$. Juan Carlos Monllau ${ }^{28}$. Gonzalo Ferrer ${ }^{29}$. Jacques Menetrey ${ }^{17}$. \\ Michael Hantes ${ }^{30}$. David Parker ${ }^{13}$. Timothy Lording ${ }^{31} \cdot$ Kristian Samuelsson $^{32,33}$. Andreas Weiler ${ }^{34}$. Soshi Uchida ${ }^{35}$. \\ Karl Heinz Frosch ${ }^{36,37}$. James Robinson ${ }^{26,38}$
}

Received: 26 August 2020 / Accepted: 13 October 2020 / Published online: 26 October 2020

(c) European Society of Sports Traumatology, Knee Surgery, Arthroscopy (ESSKA) 2020

\begin{abstract}
Purpose To establish recommendations for diagnosis, classification, treatment, and rehabilitation of posteromedial corner (PMC) knee injuries using a modified Delphi technique.

Methods A list of statements concerning the diagnosis, classification, treatment and rehabilitation of PMC injuries was created by a working group of four individuals. Using a modified Delphi technique, a group of 35 surgeons with expertise in PMC injuries was surveyed, on three occasions, to establish consensus on the inclusion or exclusion of each statement. Experts were encouraged to propose further suggestions or modifications following each round. Pre-defined criteria were used to refine item lists after each survey. The final document included statements reaching consensus in round three.

Results Thirty-five experts had a $100 \%$ response rate for all three rounds. A total of 53 items achieved over $75 \%$ consensus. The overall rate of consensus was $82.8 \%$. Statements pertaining to PMC reconstruction and those regarding the treatment of combined cruciate and PMC injuries reached $100 \%$ consensus. Consensus was reached for $85.7 \%$ of the statements on anatomy of the PMC, $90 \%$ for those relating to diagnosis, $70 \%$ relating to classification, $64.3 \%$ relating to the treatment of isolated PMC injuries, and $83.3 \%$ relating to rehabilitation after PMC reconstruction.

Conclusion A modified Delphi technique was applied to generate an expert consensus statement concerning the diagnosis, classification, treatment, and rehabilitation practices for PMC injuries of the knee with high levels of expert agreement. Though the majority of statements pertaining to anatomy, diagnosis, and rehabilitation reached consensus, there remains inconsistency as to the optimal approach to treating isolated PMC injuries. Additionally, there is a need for improved PMC injury classification.

Level of evidence Level V.
\end{abstract}

Keywords Posteromedial corner $\cdot$ Knee $\cdot$ Diagnosis $\cdot$ Treatment $\cdot$ Delphi $\cdot$ Medial collateral ligament

\section{Introduction}

Jorge Chahla

jachahla@msn.com

Extended author information available on the last page of the article
The posteromedial corner (PMC) of the knee consists of several overlapping ligaments, capsular thickenings and tendons that contribute to knee stability [2]. Injury is common [19] and compromise of the medial restraints may result in the development of valgus and rotatory laxities, increased forces on the cruciate ligaments or persistent pain and dysfunction [2, 7, 8, 24]. Although many low-grade PMC injuries 
can heal with rehabilitation alone $[10,15,16]$, some remain symptomatic following conservative treatment and may require operative intervention [19]. Similarly, determining which higher-grade PMC injuries warrant surgical repair or reconstruction can be challenging and many surgical techniques have been described.

There has been much effort to optimise intra-articular cruciate ligament reconstructions, but more recently there has been increased interest in the importance of the peripheral structures $[1,13]$ and of the need to reconstruct these, in addition to the cruciate ligaments, if they have been damaged. Despite the frequency of PMC injuries, the lack of evidence-based recommendations for diagnosis and classification and the lack of standardised treatment algorithms pose a challenge for the improved understanding and management of these injuries [2, 5, 23]. Therefore, creating a set of recommendations around both currently accepted and controversial issues in the management of PMC injuries would be of great utility to clinicians, helping to improve understanding and optimize patient outcomes.

The purpose of the current study was to develop an international consensus statement on the diagnosis, classification, treatment, and rehabilitation concepts of PMC injuries and to provide future directions for further research to address important gaps in the literature.

\section{Materials and methods}

\section{Delphi panel selection}

Four individuals (initials blinded for review.) collaborated, as a working group, to create and facilitate the development of consensus statements using a modified Delphi technique. This process (Fig. 1) was conducted under the leadership of the chair of the ESSKA Knee Collateral Ligament working group (initials blinded for review) and the consensus project leader (initials blinded for review). A total of 35 internationally recognized experts in the management of PMC injuries participated. Experts were defined as surgeons that have performed more than 50 posteromedial reconstructions and/or who have scientific interest and have published on the topic of posteromedial corner reconstruction.

\section{Statement development and item inclusion}

Statements for inclusion within the first round survey were prepared by the working group based on an analysis of three recently published systematic reviews $[2,4,7]$. A comprehensive list of statements was generated under seven categories: anatomy and biomechanics, diagnosis, classification, treatment (isolated PMC injuries), treatment (combined $\mathrm{PMC} / \mathrm{Cruciate}$ injuries), reconstruction and rehabilitation.

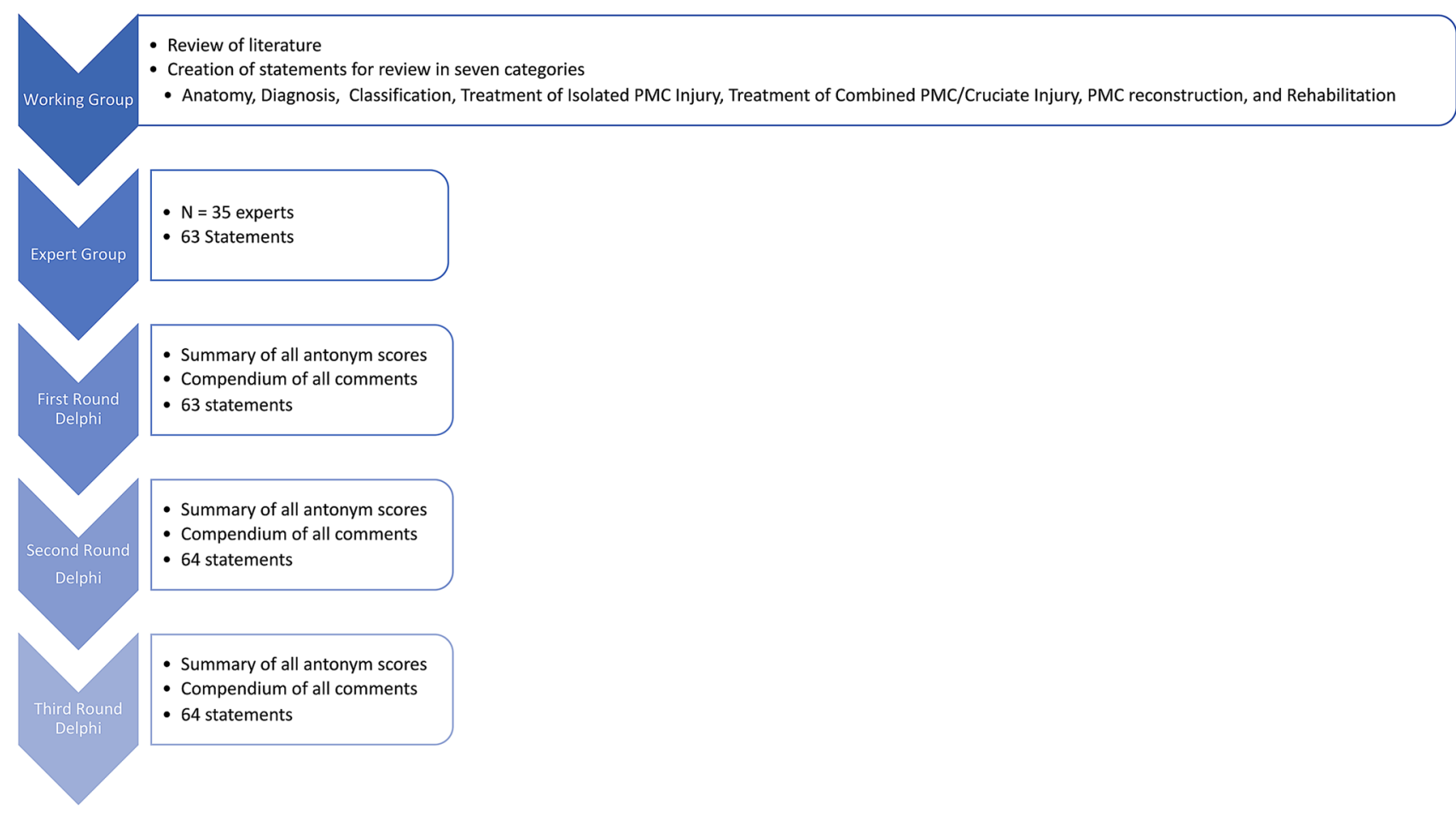

Fig. 1 Flow diagram of consensus process 
These categories were created to aid in a broad set of concepts to effectively understand and manage PMC injuries.

\section{Establishing consensus using delphi methods}

Delphi methods were used to establish group consensus on whether statements should be included in an international expert consensus document [27] relating to PMC injuries. Online surveys were generated to allow respondents to rate whether items should be included within minimum reporting requirements with five possible responses on a Likert [20] scale: "strongly agree"; "agree"; "neither agree nor disagree"; "disagree" or "strongly disagree". The survey was piloted by three experts for face validity, understanding and acceptability, resulting in minor modifications.

The PMC expert group participated in three rounds of surveys between April and August of 2020 to establish consensus on the inclusion/exclusion of each item. In addition to rating agreement through a Likert Scale, a free-text comments section was included to allow for suggestions of modifications or additional items. Pre-defined criteria were used to refine item lists after each survey. Finally, the working group compiled the final information to represent the additive opinion of the expert panel.

First-round surveys were analysed, and participants were sent an anonymized summary of the results together with a second survey. In round one, items were categorized as 'essential' and retained for round two if over $70 \%$ of respondents agreed, and fewer than $20 \%$ disagreed. Items not meeting these criteria were discarded or modified according to suggestions by responders.

The second-round survey included any new items suggested by experts in round one in addition to those deemed essential. In round two, participants were asked to rescore items and provide free-text comments. In round two, responses were analysed retaining items if over $70 \%$ of respondents agreed on their inclusion, and fewer than $20 \%$ disagreed.

Items retained after round two were considered in round three. Questionnaires were re-analysed and the cycle repeated in round three. For consensus, defined a priori, items were included in the final consensus document if over $75 \%$ of respondents agreed, and fewer than $20 \%$ disagreed in the third round Delphi survey. Agreement in $75 \%$ of participants is the most frequently specified determination of a consensus for Delphi studies [6].

\section{Results}

After screening relevant literature sources for evaluation and management strategies concerning PMC injuries, a total of 63 items were created based on a previously performed critical analysis review [2]. These items were categorized into seven groups: (1) anatomy, (2) diagnosis, (3) classification, (4) isolated PMC treatment, (5) combined PMC treatment, (6) reconstruction techniques, and (7) rehabilitation. For all three rounds, a $100 \%$ response rate was observed for all items. General results of each round are displayed in Table 1.

Round three evolved to include a total of 64 items after consideration of new items from the panel of experts. In this final round, consensus for agreement was reached for 53/64 $(82.8 \%)$. Of those not reaching consensus, one reached near agreement (74.3\% agreement). The final list of statements reaching consensus is displayed in Table 2. Statements not reaching consensus are listed in Table 3. A summary of consensus findings is outlined in the proceeding sections. Statements pertaining to treatment of combined PMC and cruciate injuries and PMC reconstruction reached $100 \%$ consensus. Consensus was reached in $85.7 \%$ of statements relating to anatomy of the PMC, $90 \%$ relating to diagnosis, $70 \%$ relating to classification, $64.3 \%$ relating to treatment of isolated PMC injuries, and $83.3 \%$ relating to rehabilitation after PMC reconstruction.

\section{Anatomy}

Understanding the anatomy of the PMC and the biomechanical roles of each structure is essential for performing PMC reconstruction with the aim of restoring near normal knee kinematics after PMC injury. To this end, the expert group agreed that the key passive restraining structures of the PMC are the superficial medial collateral ligament (sMCL), the deep MCL (dMCL), and the posterior oblique ligament (POL). In particular, the sMCL is the primary passive restraint to valgus rotation, and its rupture leads to valgus laxity at all knee flexion angles, whilst isolated rupture of the dMCL does not cause a clinically discernible increase in valgus laxity. Furthermore, experts agreed that the SMCL is the most important medial restraint to external tibial rotation.

Experts also agreed that the POL is an important restraint to internal tibial rotation while the knee is in extension. Consensus was reached regarding the role of the semimembranosus as an important dynamic restraint but there was

Table 1 Summary of results at completion of each survey round in the Delphi process to establish an expert consensus on posteromedial corner injury evaluation and management

\begin{tabular}{lllll}
\hline Delphi round & $\begin{array}{l}\text { Response } \\
\text { rate (\%) }\end{array}$ & Total items & $\begin{array}{l}\text { Items } \\
\text { reaching } \\
\text { consensus }\end{array}$ & $\begin{array}{l}\text { Modifica- } \\
\text { tions or new } \\
\text { items }\end{array}$ \\
\hline 1 & 100 & 62 & $43(69.4 \%)$ & 11 \\
2 & 100 & 64 & $51(79.7 \%)$ & 5 \\
3 & 100 & 64 & $53(82.8 \%)$ & 0 \\
\hline
\end{tabular}


Table 2 List of statements reaching consensus after Delphi round 3

Anatomy

The key passive restraining structures of the Posteromedial Corner (PMC) of the Knee are the Superficial

Oblique Ligament (POL)

The sMCL is the primary restraint to valgus rotation

Isolated rupture of the dMCL does not cause a clinically discernable increase in valgus laxity

The sMCL is the most important restraint to external tibial rotation on the medial side of the knee

The POL is an important restraint to internal tibial rotation in the extended knee

The semimembranosus is an important dynamic restraint

Diagnosis

Clinical examination, including valgus stress testing, is highly effective in diagnosing a posteromedial corner 97.1 injury

Both valgus and tibiofemoral rotation should be assessed and taken into consideration when planning treatment of posteromedial corner of the knee

Valgus laxity with the knee in slight flexion (15-30 degrees) indicates injury to the sMCL

Pronounced valgus laxity, with the knee in extension, indicates a combined injury of the sMCL and POL, and 97.1 possibly an ACL injury

A positive dial test may indicate anteromedial rotatory laxity

A strongly positive anteromedial draw test, with the knee at 90 degrees of flexion, may indicate combined

Magnetic resonance imaging should always be performed in the case of suspected grade 3 MCL injury

Valgus stress radiographs constitute an important diagnostic tool to assess the extent of an MCL injury, particularly in chronic cases ( $>6$ weeks)

Valgus stress radiographs, to assess PMC stability, are a useful assessment tool following a period of nonoperative management or following surgery

Classification

A subjective classification system based on valgus laxity findings at 0 degrees and 15-30 degrees of knee flexion (Grade $1=$ No laxity, Grade 2=Laxity at 15-30 degrees, Grade $3=$ Laxity at both 0 degrees and $15-30$ degrees) is prognostic and guides treatment

An objective classification system (e.g. based on joint-line opening on stress radiographs) is prognostic and guides treatment

Complete rupture of the POL in addition to SMCL rupture with valgus gapping in full extension is prognostic of residual valgus laxity following conservative treatment

Improved classification systems are required for posteromedial corner injuries (for example classifying grade of sMCL injury, POL injury and rotational laxities)

MRI classification of PMC injury should report on the integrity and portions (meniscofemoral and meniscotibial) of the sMCL, dMCL and POL

Grade 3 injury on MRI is represented by complete ligamentous discontinuity with laxity or waviness, suggesting disruption of all three components of the PMC (sMCL, dMCL and POL)

MRI of a chronic PMC injury will not provide information as to the extent of injury nor degree of laxity of the anatomic structures

Isolated PMC treatment

Isolated Partial ruptures of the sMCL should be treated conservatively with a range-of-motion brace

Early, immediate range of motion (0-90 degrees) within the brace should be allowed to prevent stiffness

Isolated, complete PMC ruptures that are femoral sided (meniscofemoral) or mid-substance have a more favorable outcome with conservative treatment compared to tibial sided injuries

Displaced tibial sMCL avulsions with valgus laxity are best addressed surgically

Intra-articular entrapment is an indication for acute MCL repair/reconstruction

An "MCL Stener lesion" (The distal sMCL displaced and lying superficial to the Pes Anserinus tendons) is an indication for acute MCL repair/reconstruction

The evidence for Polyethylene tape re-enforcement ("Internal Bracing") does not support its use in treatment of isolated, acute, partial sMCL injuries 
Table 2 (continued)

\begin{tabular}{|c|c|c|}
\hline Statement & $\%$ Agreement & $\%$ Disagreement \\
\hline $\begin{array}{l}\text { The evidence for Polyethylene tape re-enforcement ("Internal Bracing") does not support its use in treatment } \\
\text { of isolated, complete sMCL injuries }\end{array}$ & 91.4 & 8.6 \\
\hline \multicolumn{3}{|l|}{ Combined PMC treatment } \\
\hline $\begin{array}{l}\text { The treatment of choice for partial PMC injuries, combined with ACL rupture, is a period in a range-of- } \\
\text { motion brace before delayed, isolated ACL reconstruction }\end{array}$ & 100 & 0 \\
\hline $\begin{array}{l}\text { The treatment of choice for complete PMC injuries, combined with ACL rupture, is a period in a range-of- } \\
\text { motion brace before delayed, isolated ACL reconstruction, if medial stability is reasonably restored }\end{array}$ & 88.6 & 11.4 \\
\hline $\begin{array}{l}\text { Combined ACL rupture and tibial sMCL avulsion is an indication for early MCL repair/reconstruction and } \\
\text { ACL reconstruction }\end{array}$ & 100 & 0 \\
\hline Isolated ACL reconstruction in the presence of valgus laxity of $<3 \mathrm{~mm}$ side-to-side laxity is reasonable & 94.3 & 5.7 \\
\hline $\begin{array}{l}\text { Combined ACL, PMC reconstruction is indicated for residual medial laxity following conservative treatment } \\
\text { of the PMC injury }\end{array}$ & 100 & 0 \\
\hline $\begin{array}{l}\text { The evidence for Polyethylene tape re-enforcement ("Internal Bracing") does not support combined acute } \\
\text { "Internal Bracing" of the Medial side and ACL reconstruction for the treatment of combined, complete } \\
\text { ACL and MCL rupture }\end{array}$ & 91.4 & 8.6 \\
\hline It is reasonable to treat acute PMC injuries with complete PCL rupture with a dynamic PCL brace & 80 & 20 \\
\hline $\begin{array}{l}\text { Three ligament ruptures (KD3) involving the MCL (e.g. ACL, PCL, MCL) are best managed by early surgical } \\
\text { reconstruction of all ligaments }\end{array}$ & 94.3 & 5.7 \\
\hline \multicolumn{3}{|l|}{ Reconstruction } \\
\hline Posteromedial corner reconstructions should address both valgus and rotational laxity & 100 & 0 \\
\hline $\begin{array}{l}\text { Individual PMC structures should be reconstructed only if lax, avoiding reconstruction of structures that are } \\
\text { not damaged/lax }\end{array}$ & 100 & 0 \\
\hline $\begin{array}{l}\text { PMC reconstructions should address the anatomic deficiency based upon combined clinical examination and } \\
\text { imaging findings }\end{array}$ & 100 & 0 \\
\hline $\begin{array}{l}\text { Anatomic reconstructions with elements to reconstruct the sMCL and POL are the reconstruction of choice } \\
\text { for chronic PMC laxity }\end{array}$ & 100 & 0 \\
\hline $\begin{array}{l}\text { Long limb radiographs should be ordered, in all cases where PMC reconstruction is being considered, to } \\
\text { evaluate for the presence of valgus alignment }\end{array}$ & 97.1 & 2.9 \\
\hline $\begin{array}{l}\text { For cases of chronic PMC laxity, valgus alignment (mechanical axis alignment within the lateral compart- } \\
\text { ment) should be corrected before or with PMC reconstruction }\end{array}$ & 100 & 0 \\
\hline Ipsilateral hamstring autograft is a reasonable option for $\mathrm{PMC}$ reconstruction & 80 & 20 \\
\hline Allograft is a valid option for PMC reconstruction & 91.4 & 8.6 \\
\hline Synthetic grafts are NOT a usual first-line option for PMC reconstruction & 100 & 0 \\
\hline \multicolumn{3}{|l|}{ Rehabilitation } \\
\hline A staged rehabilitation is vital for a successful outcome & 100 & 0 \\
\hline A knee brace should be utilized after posteromedial corner reconstruction & 100 & 0 \\
\hline Early (day 1) range of motion should be implemented to avoid stiffness & 97.1 & 2.9 \\
\hline Return to sport following PMC reconstruction should be based on objective functional tests & 100 & 0 \\
\hline Return to sport following PMC reconstruction is not recommended before 6 months after surgery & 100 & 0 \\
\hline
\end{tabular}

$P M C$ Posteromedial Corner, $s M C L$ superficial medial collateral ligament, $d M C L$ deep medial collateral ligament, $P O L$ posterior oblique ligament, $A C L$ anterior cruciate ligament

disagreement as to the importance of the semitendinosus and the gracilis as dynamic restrains, with the majority (57.1\%) indicating that they felt these structures were not important.

\section{Diagnosis}

Accurate and reproducible methods to diagnosis of PMC injuries are essential to their timely treatment, especially in the context of other ligamentous injuries. Experts agreed that clinical examination, including valgus stress testing, is a highly effective method for diagnosing PMC injuries. The importance of specific clinical tests was also emphasized, such as the dial and anteromedial drawer tests, to assess for anteromedial rotary laxity.

With regard to imaging for PMC injuries, MRI was deemed a valuable tool in diagnosing acute pathology and should be requested where grade III MCL injury is suspected. Valgus stress radiographs were a commonly used diagnostic tool and particularly important for more chronic injuries. Experts agreed that these radiographs can be useful 
Table 3 List of statements failing to reach consensus after Delphi round 3

\begin{tabular}{|c|c|c|c|}
\hline & Statement & $\%$ Agreement & $\%$ Disagreement \\
\hline Anatomy & The semitendinosus and gracilis are important dynamic restraints & 42.9 & 57.1 \\
\hline Diagnosis & Ultrasound is a valuable tool in the evaluation of PMC injury & 2.9 & 97.1 \\
\hline \multirow[t]{3}{*}{ Classification } & $\begin{array}{l}\text { A subjective classification system for PMC injury recording } \mathrm{mm} \text { of joint opening } \\
\text { (Grade } 1=3-5 \mathrm{~mm} \text {, Grade } 2=6-10 \mathrm{~mm} \text {, Grade } 3>10 \mathrm{~mm} \text { ) is prognostic and } \\
\text { guides treatment }\end{array}$ & 25.7 & 74.3 \\
\hline & $\begin{array}{l}\text { A subjective classification system based on: Grade1: ligament sprained but intact, } \\
\text { Grade } 2 \text { : partial tearing with mild laxity, Grade } 3 \text { : complete tear with valgus laxity } \\
\text { is prognostic and guides treatment }\end{array}$ & 2.9 & 97.1 \\
\hline & $\begin{array}{l}\text { Existing classifications encompass the majority of the injuries and have a prognostic } \\
\text { and treatment correlation }\end{array}$ & 0 & 100 \\
\hline \multirow[t]{5}{*}{ Isolated PMC treatment } & $\begin{array}{l}\text { Isolated complete ruptures of the PMC (sMCL, dMCL and POL) can be treated } \\
\text { conservatively and successfully with a range-of-motion brace }\end{array}$ & 40 & 60 \\
\hline & $\begin{array}{l}\text { For isolated, complete PMC injuries, it is not necessary to initially lock the range-of- } \\
\text { motion brace in extension / slight flexion for a short period (e.g. } 2 \text { weeks) }\end{array}$ & $74.3^{*}$ & 25.7 \\
\hline & NSAIDs do not impair healing of PMC injuries & 62.9 & 37.1 \\
\hline & PRP injections augment the healing of PMC injuries & 5.7 & 94.3 \\
\hline & $\begin{array}{l}\text { Peri ligamentous corticosteroid injection is reasonable for ongoing medial pain fol- } \\
\text { lowing conservative treatment }\end{array}$ & 48.5 & 51.5 \\
\hline Rehabilitation & $\begin{array}{l}\text { Following PMC reconstruction, patients should remain non-weight-bearing / toe- } \\
\text { touch weight-bearing for a minimum of six weeks }\end{array}$ & 34.3 & 65.7 \\
\hline
\end{tabular}

$P M C$ Posteromedial Corner, $s M C L$ superficial medial collateral ligament, $d M C L$ deep medial collateral ligament, $P O L$ posterior oblique ligament, $A C L$ anterior cruciate ligament

*Reached near consensus

to determine residual laxity following conservative management and also to assess graft/repair function postoperatively.

\section{Classification}

It was recognised that valgus laxity should be assessed with the knee in 15 to 30 degrees flexion and with the knee in extension and joint-line opening can be objectively assessed with stress radiographs at both flexion angles. The degree of opening is prognostic and guides treatment. In particular, combined rupture of the sMCL and POL causing significant valgus laxity in the extended knee is prognostic of residual valgus laxity following conservative treatment. There, however, remains a lack of comprehensive and prognostic classification systems to evaluate injury extent and guide treatment. To this end, experts agreed that it is important to develop improved classification systems for PMC injuries. Such classifications should incorporate MRI findings of the integrity and portions of the SMCL, dMCL, and POL involved and consider injury chronicity.

\section{Treatment of isolated PMC Injury}

The timing and extent of PMC involvement influence the management of these injuries. There was broad consensus that isolated partial ruptures of the sMCL should be treated conservatively with a range-of-motion (ROM) brace. There was disagreement as to whether isolated grade 3 PMC injuries (where sMCL, dMCL. and POL are injured) can be treated conservatively in a brace. However, most agreed that for isolated, complete PMC injuries, it is not necessary to initially lock the range-of-motion brace in extension/slight flexion for a short period (74.3\% agreement), although this statement did not reach the consensus threshold of $75 \%$. Experts agreed that factors necessitating acute surgical intervention included both tibial and femoral bony avulsions, intra-articular entrapment, and identification of an MCL "Stener lesion" (where ruptured layer 2 lies superficial to the layer 1 [sartorius fascia]) [3]. Experts felt strongly that there was a lack of evidence supporting the treatment of both partial and complete sMCL injuries with polyethylene tape re-enforcement. Experts felt strongly that PRP injections do not augment MCL healing. There was no consensus as to whether NSAIDs may impair PMC healing (62.9\% of experts disagreed) although it was discussed that this may be dose-dependent. There is no consensus on the role of peri-ligamentous corticosteroid injection for persistent pain.

\section{Treatment of combined PMC and cruciate injury}

Experts agreed that the treatment of choice for both partial and complete PMC injuries, when combined with an ACL rupture, is a period in a ROM brace prior to delayed ACL reconstruction. There was agreement that isolated ACL reconstruction 
was reasonable in the presence of persistent valgus laxity of $<3 \mathrm{~mm}$ (side-to-side difference). If increased medial laxity is observed following the period of conservative treatment, then combined ACL and PMC reconstruction is indicated. Similar to isolated PMC injury, experts recommended against the use of polyethylene tape reinforcement of the medial side for combined injuries. When ACL rupture is associated with a tibial SMCL avulsion, ACL reconstruction should not be delayed and the MCL should be simultaneously and acutely repaired/reconstructed. For combined acute PMC injury with PCL rupture, experts agreed that it is reasonable to treat these injuries with a dynamic PCL brace. Three ligament ruptures (ACL, PCL, and MCL) should also be managed with acute reconstruction.

\section{Reconstruction techniques}

Experts strongly agreed that the goal of PMC reconstruction should be to address both valgus and rotational laxity and that structures should only be reconstructed if found to be compromised with resultant laxity. Consequentially, reconstruction techniques should address the anatomic and biomechanical deficiencies observed during clinical examination and review of imaging (MRI and stress radiographs). For chronic PMC laxity, anatomic reconstruction of the SMCL and POL is recommended. If valgus alignment on long-limb radiographs is observed in the context of chronic PMC laxity, alignment should be corrected prior to, or simultaneously, with PMC reconstruction. Use of allografts or ipsilateral hamstring autografts is a reasonable option for PMC reconstruction, though synthetic grafts are not a usual first-line option.

\section{Rehabilitation}

Rehabilitation must be considered in the context of the degree of injury and type of treatment performed, though experts agreed on certain practices regardless of these factors. All experts agreed that a staged rehabilitation is imperative for a successful outcome after PMC reconstruction and that a knee brace should be utilized in all cases. Early range of motion should begin on the first post-operative day one to avoid knee stiffness. For PMC injuries in the athlete, return to sport should be based on objective functional tests and is not recommended before postoperative month six (Fig. 2). There was no consensus as to post-operative weight-bearing status.

\section{Discussion}

The main finding of the current study was the high level of consensus amongst international experts on the management of injuries to the PMC reached on the majority of statements
$(82.8 \%)$. Broad agreement was found regarding the anatomy, diagnosis, reconstruction, and rehabilitation practices for PMC injuries.

The complex anatomy of the PMC has also been highlighted and described by numerous studies. [7, 25, 26]. Successful treatment of injuries of the PMC of the knee requires a comprehensive understanding of the structures, their interactions and biomechanical properties essential for stability and normal knee kinematics. Experts agreed that key passive restraining structures are the SMCL, dMCL, and the POL. The importance of these structures, with regard to both valgus and rotational stability, is recognised and there is a risk of force being transferred to the cruciates as a result of PMC compromise [2]. For reconstruction, it is essential that the anatomy of these structures is understood as failure to do so may result in short tunnels or collisions between drilled tunnels, especially when combined with cruciate ligament reconstruction [12].

The current expert consensus highlighted the importance of a diagnostic approach and classification to guide appropriate treatment. Specifically, there was considerable agreement in the assessment of valgus stress testing in flexion and extension, assessing for tibiofemoral rotation, and the use of the dial and anteromedial drawer tests as important components of the physical exam. MRI may be used to characterize high-grade PMC injuries and identify concomitant soft tissue or cruciate injuries but experts also highlighted the importance of obtaining long-limb radiographs to assess for valgus knee alignment and valgus stress radiographs to assess for extent of injury, particularly in chronic cases. These recommendations are in accordance with Cinque et al. [2] who also emphasized that valgus stress radiographs and MRI are the two primary imaging modalities for diagnosis of PMC injuries. House et al. [14] also reported the benefits of using MRI for detailing the structures of the PMC and its implications in management and prognosis, highlighting the intimate relationships of these structures, which are best visualized on MRI. Geiger et al. [11] reported that MRI offers an accurate diagnostic tool for these injuries and that it is imperative to evaluate the PMC using this modality prior to surgical intervention as it may avoid complications resulting from occult injury and may provide additional insight for preoperative planning. Despite relative consensus among experts and in the literature regarding PMC injury diagnosis, classification systems for prognosis and treatment remain lacking. The expert consensus highlighted the need for an improved classification system based on injury severity and rotational laxity, which should be the subject of future studies to optimize the transition between evaluation and treatment as well as postoperative outcomes.

Isolated and combined PMC injuries often necessitate different approaches to treatment although there was consensus that the treatment of choice for both isolated and combined 
Fig. 2 Stacked leaning bar chart representing breakdown in agreement levels in the third round Delphi survey. Bars to the left of the $Y$ axis indicate disagreement with bars to the right indicating agreement

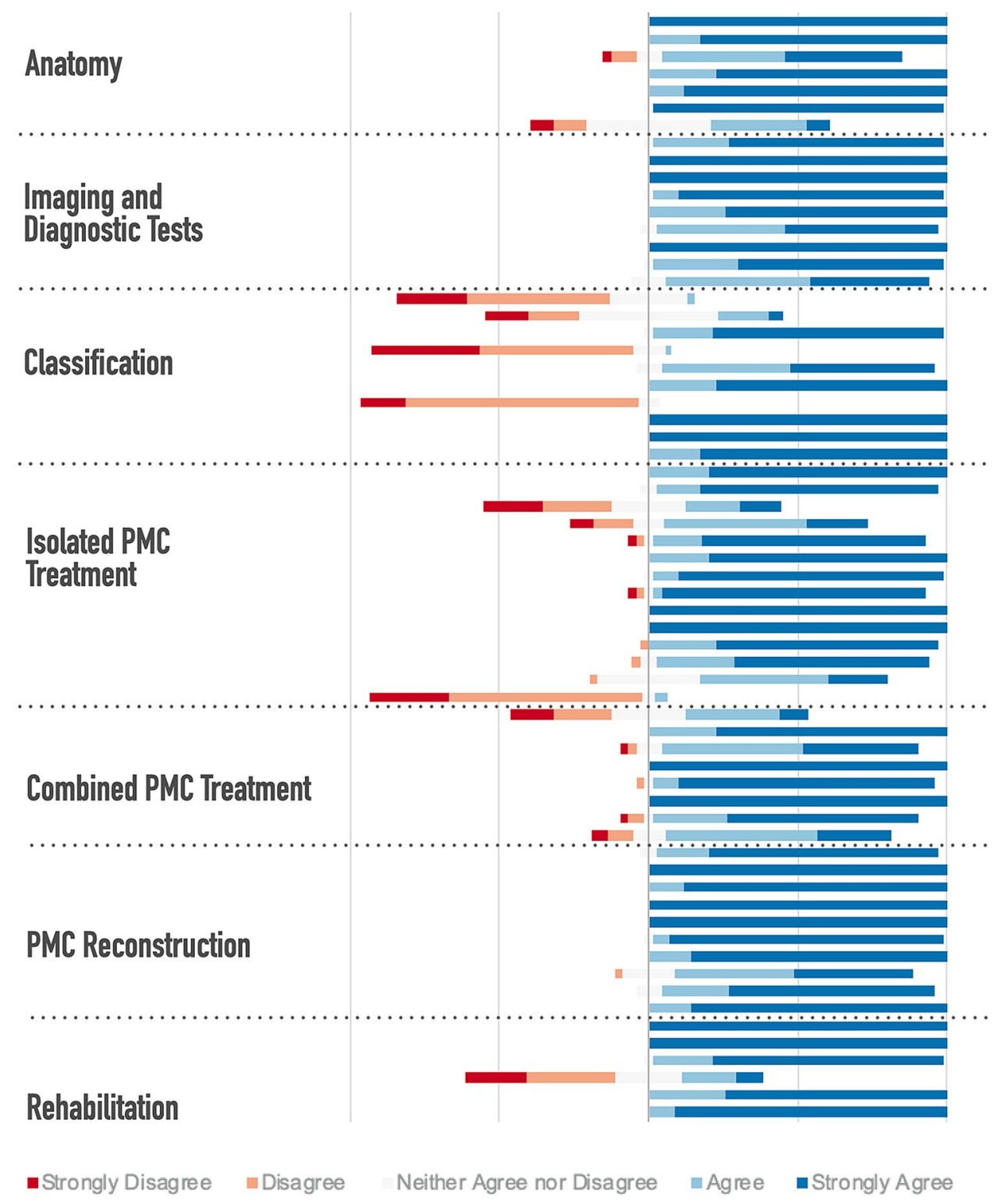

PMC injuries is with a period of conservative treatment in a ROM brace, with later cruciate reconstruction as required. There was a broad consensus for acute surgical treatment when patients present with displaced sMCL avulsion injuries, the presence of an MCL "Stener lesion" or intra-articular entrapment. There was agreement that multi-ligament injuries are best managed by early surgical reconstruction. Recent literature has suggested that appropriate treatment of the PMC in multi-ligament injuries is essential to prevent failure associated with instability conferred by other damaged structures [29]. Whilst there are many studies in the literature describing the anatomy of the PMC and many reconstruction techniques described, there is a relative lack of clinical outcome studies. While few studies have reported on non-anatomic PMC reconstruction [17, 21], Laprade and Wijdicks [19] reported the outcomes of 28 patients who underwent anatomic MCL reconstruction and POL reconstruction with cruciate ligament reconstruction. The authors found that all patients had resolution of side-to-side instability at a minimum of 2 years postoperatively, while the mean International Knee Documentation Committee score improved from 44 to 76 points. Furthermore, valgus stress radiographs demonstrated improvements from $6.3-\mathrm{mm}$ to 1.3-mm side-to-side difference and all patients had $<3-\mathrm{mm}$ joint space widening on valgus stress radiographs. However, future studies are warranted to confirm the consensus statements generated in our study given the paucity of literature characterizing the long-term outcomes of anatomic PMC reconstruction.

Consensus on rehabilitation practices was observed for all but one statement, where experts disagreed on the need for patients to be toe-touch or non-weight-bearing after PMC 
reconstruction. Interestingly, few studies have advocated non-weight-bearing or partially-weight-bearing after PMC construction, which is in accordance with this disagreement $[18,28,30]$. However, there was agreement for the use of staged rehabilitation, use of a ROM knee brace, early mobilization, and protocols for return to sport stressing the importance of both functional goal attainment as well as time. This consensus is in accordance with literature suggesting that early protected range of motion and rehabilitation should be performed to avoid stiffness [19, 22]. Furthermore, the literature supports the consensus of return to sport after six months of rehabilitation assuming appropriate progression and achievement of strength and function milestones [9, 21].

The current study which established a set of consensus statements generated by a panel of 35 international experts has strengths which include: (1) the $100 \%$ response rate across all three rounds, (2) the use of a Delphi method which overcomes limitations inherent in group-based processes including subject anonymity and inability to meet face-toface, especially in the current environment of the COVID-19 pandemic at the time of this writing; and (3) the ability to modify, refine and add statements based on the experiences and observations of these experts. This expert consensus helps define current best practice for the evaluation and management of PMC injuries. It also highlights areas of disagreement, such as in injury classification and weight-bearing protocols after PMC treatment (Table 3), allowing clinicians who treat PMC injuries to be aware of issues of contention. The lack of accord on some subjects indicates the need for additional research so that patient care can be optimized.

Though this study exhibits these strengths, certain limitations must also be considered. Ultimately, these statements represent the opinions and personnel experiences of experts despite being generated from the literature and published evidence. Moreover, differences in practices and resources may preclude these statements from being generalizable, though it is the goal of this study to induce consistency and change across practices to optimize the diagnosis and treatment of PMC injuries. Future research is warranted to develop objective classification systems to optimize diagnosis and treatment and to better understand the outcomes of PMC treatment based on treatment approach.

\section{Conclusion}

A modified Delphi technique was applied to generate an expert consensus statement concerning the diagnosis, classification, treatment, and rehabilitation practices for PMC injuries of the knee with high levels of expert agreement. Though the majority of statements pertaining to anatomy, diagnosis, and rehabilitation reached consensus, there remains inconsistency in optimal approach to treating isolated PMC injuries and need for more accurate and prognostic classification systems.

Author contributions JC, RFL, AG, JR: created and facilitated development of consensus statements; Jorge Chahla and James Robinson supervised the project and revised the final manuscript. KK: survey data collection, writing of initial manuscript. MC, PG, BB, NP, ML, RA, BF, FM, LR, JK, VD, DT, ZG, NG, SZ, LE, ML, RM, RVB, CB, SV, JCM, GF, JM, MH, DP, TL, KS, AW, SU, KHF: expert group respondents.

Funding No funding was received for this work.

\section{Compliance with ethical standards}

Conflict of interest The authors declare no conflict of interest in relation to the subject of work.

Ethical approval This study was exempt from institutional board approval.

\section{References}

1. Chahla J, Murray IR, Robinson J, Lagae K, Margheritini F, Fritsch B et al (2019) Posterolateral corner of the knee: an expert consensus statement on diagnosis, classification, treatment, and rehabilitation. Knee Surg Sports Traumatol Arthrosc 27:2520-2529

2. Cinque ME, Chahla J, Kruckeberg BM, DePhillipo NN, Moatshe G, LaPrade RF (2017) Posteromedial corner knee injuries: diagnosis, management, and outcomes: a critical analysis review. JBJS Rev $5:$ e4

3. Corten K, Hoser C, Fink C, Bellemans J (2010) Case reports: a Stener-like lesion of the medial collateral ligament of the knee. Clin Orthop Relat Res 468:289-293

4. DeLong JM, Waterman BR (2015a) Surgical repair of medial collateral ligament and posteromedial corner injuries of the knee: a systematic review. Arthroscopy 31(2249-2255):e2245

5. DeLong JM, Waterman BR (2015b) Surgical techniques for the reconstruction of medial collateral ligament and posteromedial corner injuries of the knee: a systematic review. Arthroscopy 31(2258-2272):e2251

6. Diamond IR, Grant RC, Feldman BM, Pencharz PB, Ling SC, Moore AM et al (2014) Defining consensus: a systematic review recommends methodologic criteria for reporting of Delphi studies. J Clin Epidemiol 67:401-409

7. Dold AP, Swensen S, Strauss E, Alaia M (2017) The posteromedial corner of the knee: anatomy, pathology, and management strategies. J Am Acad Orthop Surg 25:752-761

8. Engebretsen L, Lind M (2015) Anteromedial rotatory laxity. Knee Surg Sports Traumatol Arthrosc 23:2797-2804

9. Fanelli GC, Harris JD (2006) Surgical treatment of acute medial collateral ligament and posteromedial corner injuries of the knee. Sports Med Arthrosc Rev 14:78-83

10. Fetto JF, Marshall JL (1978) Medial collateral ligament injuries of the knee: a rationale for treatment. Clin Orthop Relat Res 132:206-218

11. Geiger D, Chang E, Pathria M, Chung CB (2013) Posterolateral and posteromedial corner injuries of the knee. Radiol Clin North Am 51:413-432 
12. Gelber PE, Masferrer-Pino A, Erquicia JI, Abat F, Pelfort X, Rodriguez-Baeza A et al (2015) Femoral tunnel drilling angles for posteromedial corner reconstructions of the knee. Arthroscopy 31:1764-1771

13. Getgood A, Brown C, Lording T, Amis A, Claes S, Geeslin A et al (2019) The anterolateral complex of the knee: results from the International ALC Consensus Group Meeting. Knee Surg Sports Traumatol Arthrosc 27:166-176

14. House CV, Connell DA, Saifuddin A (2007) Posteromedial corner injuries of the knee. Clin Radiol 62:539-546

15. Jacobson KE, Chi FS (2006) Evaluation and treatment of medial collateral ligament and medial-sided injuries of the knee. Sports Med Arthrosc Rev 14:58-66

16. Kannus P (1988) Long-term results of conservatively treated medial collateral ligament injuries of the knee joint. Clin Orthop Relat Res 226:103-112

17. Kim SJ, Lee DH, Kim TE, Choi NH (2008) Concomitant reconstruction of the medial collateral and posterior oblique ligaments for medial instability of the knee. J Bone Joint Surg Br 90:1323-1327

18. Koga H, Muneta T, Yagishita K, Ju YJ, Sekiya I (2012) Surgical management of grade 3 medial knee injuries combined with cruciate ligament injuries. Knee Surg Sports Traumatol Arthrosc 20:88-94

19. Laprade RF, Wijdicks CA (2012) The management of injuries to the medial side of the knee. J Orthop Sports Phys Ther 42:221-233

20. Likert R (1932) A technique for the measurement of attitudes. Arch Psych 22:55-56

21. Lind M, Menhert F, Pedersen AB (2009) The first results from the Danish ACL reconstruction registry: epidemiologic and 2 year follow-up results from 5,818 knee ligament reconstructions. Knee Surg Sports Traumatol Arthrosc 17:117-124

22. Logan CA, O'Brien LT, LaPrade RF (2016) Post operative rehabilitation of grade iii medial collateral ligament injuries: evidence based rehabilitation and return to play. Int J Sports Phys Ther 11:1177-1190

23. Lubowitz JH (2015) Editorial commentary: medial collateral ligament and posteromedial corner reconstruction techniques vary and indications are not clear. Arthroscopy 31:2273

24. Offerhaus C, Balke M, Arner JW, Musahl V, Hoher J (2018) Reefing of the posteromedial capsule in anteromedial rotatory instability. Arthrosc Tech 7:e547-e551

25. Robinson JR, Sanchez-Ballester J, Bull AM, Thomas Rde W, Amis AA (2004) The posteromedial corner revisited. An anatomical description of the passive restraining structures of the medial aspect of the human knee. J Bone Joint Surg Br 86:674-681

26. Sims WF, Jacobson KE (2004) The posteromedial corner of the knee: medial-sided injury patterns revisited. Am J Sports Med 32:337-345

27. Stacey DBC, Barry MJ, Col NF, Eden KB, Holmes-Rovner M, et al. Decidion aids for people facing health treatment or screening decisions. Cochrane Database Syst Rev 2011;(10)CD001431

28. Tardy N, Boisrenoult P, Teissier P, Steltzlen C, Beaufils P, Pujol N (2017) Clinical outcomes after multiligament injured knees: medial versus lateral reconstructions. Knee Surg Sports Traumatol Arthrose 25:524-531

29. Tibor LM, Marchant MH Jr, Taylor DC, Hardaker WT Jr, Garrett WE Jr, Sekiya JK (2011) Management of medial-sided knee injuries, part 2: posteromedial corner. Am J Sports Med 39:1332-1340

30. Vaughn ZD, Schmidt J, Lindsey DP, Dragoo JL (2009) Biomechanical evaluation of a 1-stage revision anterior cruciate ligament reconstruction technique using a structural bone void filler for femoral fixation. Arthroscopy 25:1011-1018

Publisher's Note Springer Nature remains neutral with regard to jurisdictional claims in published maps and institutional affiliations.

\section{Affiliations}

Jorge Chahla ${ }^{1} \cdot$ Kyle N. Kunze ${ }^{2} \cdot$ Robert F. LaPrade $^{3} \cdot$ Alan Getgood $^{4} \cdot$ Moises Cohen $^{5} \cdot$ Pablo Gelber $^{6,7}$. Björn Barenius $^{8} \cdot$ Nicolas Pujol $^{9,10} \cdot$ Manual Leyes $^{11} \cdot$ Ralph Akoto $^{12} \cdot$ Brett Fritsch $^{13} \cdot$ Fabrizio Margheritini $^{14}$. Leho Rips ${ }^{15}$. Jakub Kautzner ${ }^{16}$. Victoria Duthon ${ }^{17}$. Danilo Togninalli ${ }^{18}$. Zanon Giacamo ${ }^{19}$. Nicolas Graveleau ${ }^{20}$. Stefano Zaffagnini ${ }^{21}$. Lars Engbretsen ${ }^{22} \cdot$ Martin Lind $^{23} \cdot$ Rodrigo Maestu $^{24} \cdot$ Richard Von Bormann $^{25}$. Charles Brown ${ }^{26}$. Silvio Villascusa ${ }^{27}$. Juan Carlos Monllau ${ }^{28}$. Gonzalo Ferrer ${ }^{29}$. Jacques Menetrey ${ }^{17}$. Michael Hantes ${ }^{30}$. David Parker ${ }^{13}$. Timothy Lording ${ }^{31} \cdot$ Kristian Samuelsson $^{32,33}$. Andreas Weiler ${ }^{34}$. Soshi Uchida ${ }^{35}$. Karl Heinz Frosch ${ }^{36,37}$. James Robinson ${ }^{26,38}$

1 Division of Sports Medicine, Department of Orthopaedic Surgery, Rush University Medical Center, 1611 W. Harrison Street Suite 300, Chicago, IL 60612, USA

2 Department of Orthopaedic Surgery, Hospital for Special Surgery, New York, NY, USA

3 Twin Cities Orthopaedics, Edison, MN, USA

4 Fowler Kennedy Sports Medicine Clinic, London, ON, Canada

5 Universidade Federal de São Paulo, São Paulo, SP, Brazil

6 Department of Orthopaedic Surgery, Hospital de La Sta Creu I Sant Pau, Universitat Autònoma de Barcelona, Barcelona, Spain
7 ICATME-Hospital Universitari Dexeus, Universitat Autònoma de Barcelona, Barcelona, Spain

8 Stockholm South Hospital, Karolinska Institutet, Stockholm, Sweden

9 Centre Hospitalier de Versailles, Le Chesnay, France

10 Oslo University Hospital, Oslo, Norway

11 Clínica CEMTRO, Madrid, Spain

12 Asklepios Klinik St. Georg, Chirurgisch Traumatologisches Zentrum, Hamburg, Germany

13 Sydney Orthopaedic Research Institute, Sydney, Australia

14 University of Rome Foro Italico, Rome, Italy 
15 Sports Traumatology Center, Sports Medicine and Rehabilitation Clinic, Tartu University Hospital, Tartu, Estonia

16 University Hospital Motol, Prague, Czech Republic

17 University Hospital of Geneva, Geneva, Switzerland

18 Ars Ortopedica, Clinical Ars Medica, Gravesano, Switzerland

19 University of Pavia, IRCCS Policlinico San Matteo, Pavia, Italy

20 Clinique du Sport de Bordeaux, Mérignac-Bordeaux, France

21 Istituto Ortopedico Rizzoli - University of Bologna, Bologna, Italy

22 Oslo Sports Trauma Research Center, Oslo, Norway

23 Aarhus University Hospital, Aarhus, Denmark

24 Centro de Tratamiento de Enfermedades Articulares, Buenos Aires, Argentina

25 Cape Town Sports \& Orthopaedic Clinic, Cape Town, South Africa

26 International Knee and Joint Centre, Abu Dhabi, UAE
Hospital La Fraternidad Habana, Madrid, Spain

28 Parc de Salut Mar, UAB, Barcelona, Spain

29 Hospital Clinico Mutual de Seguridad, Santiago, Chile

30 University of Thessalia, Larissa, Greece

31 Melbourne Orthopaedic Group, Melbourne, Australia

Department of Orthopaedics, Sahlgrenska University Hospital, Sahlgrenska Academy, Gothenburg University, Gothenburg, Sweden

33 Nanometer Structure Consortium, Lund University, Lund, Sweden

34 Zentrum Für Spezielle Gelenkchirurgie, Berlin, Germany

35 Wakamatsu Hospital, University of Occupational and Environmental Health, Kitakyushu, Japan

36 University Medical Center Hamburg-Eppendorf, Hamburg, Germany

37 Asklepios Clinic St. Georg, Hamburg, Germany

38 Avon Orthopaedic Centre, Bristol, UK 